\title{
Field trial to evaluate the effect of an intranasal respiratory vaccine protocol on calf health, ultrasonographic lung consolidation, and growth in Holstein dairy calves
}

\author{
T. L. Ollivett, ${ }^{*}$ K. E. Leslie,† T. F. Duffield,† D. V. Nydam,‡ J. Hewson,§ J. Caswell,\# P. Dunn,† \\ and D. F. Keltont ${ }^{1}$ \\ *Department of Medical Sciences, School of Veterinary Medicine, University of Wisconsin, Madison 53706 \\ †Department of Population Medicine, Ontario Veterinary College, University of Guelph, Guelph, ON N1G 2W1, Canada \\ $\ddagger D$ pepartment of Population Medicine and Diagnostic Sciences, College of Veterinary Medicine, Cornell University, Ithaca, NY 14850 \\ $\S$ Department of Clinical Studies, and \\ \#Department of Pathobiology, Ontario Veterinary College, University of Guelph, Guelph, ON N1G 2W1, Canada
}

\begin{abstract}
The objective of this field trial was to evaluate the effect of a vaccine protocol using a commercially available trivalent vaccine designed for intranasal use. Experimental challenge studies have demonstrated varying efficacies of vaccines administered via the intranasal route. A total of 468 calves from 3 herds were enrolled and randomized into 3 treatment groups (positive control, $\mathrm{PC}, \mathrm{n}=211$; intranasal vaccine, $\mathrm{IN}, \mathrm{n}=215$; negative control, $\mathrm{NC}, \mathrm{n}=42$ ) and followed for 8 to $12 \mathrm{wk}$. The PC consisted of one dose of commercially available multivalent injectable vaccine against bovine respiratory syncytial virus, infectious bovine rhinotracheitis, parainfluenza 3 , and bovine viral diarrhea administered subcutaneously at $6 \mathrm{wk}$ of age. The IN was administered at enrollment and 6 wk of age, and contained antigen against bovine respiratory syncytial virus, infectious bovine rhinotracheitis, and parainfluenza 3 . The NC was sterile saline administered intranasally and subcutaneously at enrollment and $6 \mathrm{wk}$ of age. Clinical illness was assessed using systematic respiratory scoring, and thoracic ultrasonography was used to identify the lung consolidation associated with pneumonia. Rib fractures were identified in $6 \%$ of calves, and an association was observed between rib fractures and calving ease. Overall, $54 \%$ of the calves had at least one episode of an abnormal respiratory score (ILL). Vaccination protocol did not affect the occurrence of ILL. Similarly, $54 \%$ of the calves had at least one episode of lung consolidation $\geq 3 \mathrm{~cm}(\mathrm{CON})$. Vaccine protocol affected the odds of CON. The odds of CON in PC were 1.63 (95\% confi-
\end{abstract}

Received December 11, 2017.

Accepted May 1, 2018.

${ }^{1}$ Corresponding author: dkelton@uoguelph.ca dence interval: $1.04-2.56)$ times the odds of CON in IN, and 0.38 (95\% confidence interval: $0.16-0.93)$ times the odds of CON in NC. The odds of CON in IN were 0.23 (95\% confidence interval: $0.09-0.59$ ) times the odds of CON in NC. The outcomes ILL and CON were associated; however, the measure of agreement was only fair $($ kappa $=0.38)$. Multivariable linear regression revealed an interaction between vaccine protocol and herd on average daily gain (ADG); therefore, these data were stratified. In herd $1, \mathrm{IN}(0.53 \pm 0.03 \mathrm{~kg} / \mathrm{d})$ decreased ADG compared with $\mathrm{PC}(0.63 \pm 0.03 \mathrm{~kg} / \mathrm{d})$. In herd 2, IN increased ADG $(0.41 \pm 0.03 \mathrm{~kg} / \mathrm{d})$ compared with $\mathrm{PC}(0.38 \pm 0.03 \mathrm{~kg} / \mathrm{d})$. In contrast, none of the protocols affected ADG at herd 3. In conclusion, this commercially available trivalent IN vaccine protocol did not alter the incidence of ILL, reduced the risk of lung lesions associated with pneumonia, and improved the ADG of the calves in one of the commercial study herds.

Key words: bovine respiratory disease, intranasal vaccine, thoracic ultrasound

\section{INTRODUCTION}

Calves are born agammaglobulinemic and must ingest maternal colostrum for immune support. Although maternal transfer of antibody to the newborn calf provides many great benefits (Faber et al., 2005), high levels of maternal antibodies are associated with delayed antibody production by the neonate, as well as selective inhibition of lymphocyte responses (Tizard, 2013). The potential for maternal blockade has caused concern regarding the practice of early life vaccination to prevent bovine respiratory disease (BRD), as maternal antibodies can be present for up to 6 mo of age (Menanteau-Horta et al., 1985). Vaccination to reduce the incidence of BRD would be beneficial, as BRD is a 
leading cause of morbidity and mortality in preweaned dairy calves (Windeyer et al., 2014).

It has been established that 3- to 8-d-old Holstein calves are capable of mounting a mucosal immune response, even when maternal antibodies are present (Hill et al., 2012). Over the last 10 yr, reports have been inconsistent regarding the potential for intranasal vaccination to protect young dairy calves, regardless of maternal antibody status, from infection with bovine respiratory syncytial virus (BRSV) and parainfluenza $3\left(\mathbf{P I}_{3}\right)$. In one study, calves between 3 and $8 \mathrm{~d}$ of age were vaccinated intranasally with a product intended for subcutaneous administration, and challenged with BRSV $21 \mathrm{~d}$ later, or at $4.5 \mathrm{mo}$ of age (Ellis et al., 2010). After the delayed challenge at $4.5 \mathrm{mo}$, seropositive calves had wider temperature fluctuations compared with seronegative calves; however, no differences were detected in other health parameters. Seronegative calves that were vaccinated intranasally with a low dose of BRSV antigen, and challenged with BRSV $21 \mathrm{~d}$ later, had less extensive lung lesions at postmortem examination, even though no differences in clinical scores or mortality were noted (Ellis et al., 2010). Intranasal vaccination in 3 - to 8 -d-old calves resulted in improved partial pressure of oxygen, fewer lung lesions, and lower mortality rate than in unvaccinated calves, when they were challenged with BRSV at $9 \mathrm{wk}$, but not $14 \mathrm{wk}$, indicating that the duration of immunity is short (Ellis et al., 2013). Additionally, 2 doses of monovalent injectable BRSV product used intranasally resulted in complete protection from clinical disease, one dose resulted in minimal clinical signs, whereas unvaccinated calves experienced severe clinical signs (Ellis et al., 2007).

The previously mentioned challenge studies often found that IN calves had less extensive lesions associated with lung consolidation than control calves despite the lack of observable clinical changes. In the beef industry, several reports suggest that evaluation of lung lesions at harvest may be a more accurate means of documenting BRD than clinical observations (Wittum et al., 1996; Thompson et al., 2006; White and Renter, 2009). Unfortunately, documenting lung lesions directly requires euthanasia, which often limits the size of study populations. As an alternative, thoracic ultrasonography (TUS) can be performed quickly and provides an accurate ante-mortem assessment of lung health (Rabeling et al., 1998; Ollivett et al., 2013, 2015). When lung consolidation identified by TUS was confirmed by the gold standard postmortem examination, TUS manifested a sensitivity of 85 and $94 \%$ in chronic clinical (Rabeling et al., 1998) and acute subclinical (Ollivett et al., 2015) cases, respectively; and specificity was 98 and $100 \%$, in chronic clinical and acute subclinical cases, respectively. Additionally, Buczinski et al. (2015) using
Bayesian latent class analysis determined the sensitivity and specificity to be 79.4 and $93.9 \%$, respectively.

The primary objective of this randomized controlled field trial was to evaluate the effect of an intranasal vaccine protocol on the health of young Holstein dairy calves. The secondary objectives were to evaluate the effect of this vaccine on ultrasonographic lung consolidation and growth.

\section{MATERIALS AND METHODS}

\section{Animals and Facilities}

This study was carried out on 3 dairies in southwestern Ontario, Canada, between January and December 2012. Two of these herds were the Elora (herd 1) and Ponsonby (herd 3) Dairy Research Centres associated with the University of Guelph. The third dairy was a privately owned commercial herd (herd 2). Each herd was visited twice a week (all herds) or 3 times a week during periods of high enrollment (herd 2 only) to enroll calves twice a week. Male and female Holstein calves were enrolled between 3 to $6 \mathrm{~d}$ of age into 3 groups according to vaccine protocol (positive control, PC; intranasal vaccine, IN; and negative control, NC) and followed for 8 to $12 \mathrm{wk}$. A birth record was filled out by the dairy producer after the birth of each calf. The PC (Bovi-Shield Gold 5, Zoetis, New York, NY) consisted of one dose of commercially available multivalent injectable vaccine against BRSV, infectious bovine rhinotracheitis, $\mathrm{PI}_{3}$, and bovine viral diarrhea administered by subcutaneous injection at 6 wk of age. The IN (Inforce 3, Zoetis) was administered twice (first dose: 3 to $6 \mathrm{~d}$ of age; second dose: 6 wk of age) and contained BRSV, infectious bovine rhinotracheitis, and $\mathrm{PI}_{3}$ antigens. The NC, sterile saline, was administered both intranasally and subcutaneously twice (first dose: 3 to $6 \mathrm{~d}$ of age; second dose: $6 \mathrm{wk}$ of age). All doses of IN were administered via a single-use plastic nasal cannula (Zoetis) into one nostril. All injections were administered subcutaneously in the neck. Treatments were administered by members of the research team not involved in respiratory scoring or TUS. Different randomization methods were used to assign calves to the 3 treatment groups at each herd because of differences in barn design. Treatment groups were housed in separate areas to prevent contamination of PC and $\mathrm{NC}$ calves from the potential nasal virus shedding by IN calves following vaccination. Weight, respiratory score (RS), and TUS observations were performed by the principal investigator (TLO) who was blinded to treatment throughout all data collection. This study was conducted with the approval of the University of Guelph's Animal Care Committee (AUP \#11R110). 


\section{Herd-Specific Calf Management}

In herd 1 (lactating cows, $\mathrm{n}=150$ ), calves were fed $4 \mathrm{~L}$ of single source colostrum within $24 \mathrm{~h}$ of birth. Calves were housed in either individual stalls within an enclosed room with a mechanical ventilation system, or outside tethered to individual plastic hutches. Calves were fed $6 \mathrm{~L}$ of whole unpasteurized milk per day until approximately $6 \mathrm{wk}$ of age, at which point they were gradually weaned and moved to group housing by $8 \mathrm{wk}$ of age. Free choice water and calf starter were available beginning at $3 \mathrm{~d}$ of age. Prior to the start of the study, rotating treatment groups were assigned to each room (enclosed nurseries, $\mathrm{n}=3$; outdoor "hutch room," $\mathrm{n}$ $=1$ ) by drawing the protocol name from a hat with replacement. Four cycles were drawn for each room to accommodate the number of calves for the anticipated duration of the study. Each room was filled with 8 to 10 calves over a 2 -wk period. Calves were housed together according to birth order regardless of sex. Each room was cleaned, disinfected, and allowed to sit empty for approximately $1 \mathrm{wk}$ before new calves were added.

In herd 2 (lactating cows, $\mathrm{n}=650$ ), single source colostrum (2 L) was offered by bottle within $30 \mathrm{~min}$ of birth. An additional $4 \mathrm{~L}$ was offered in 2 separate feedings over the $24 \mathrm{~h}$ following birth. Calves were fed $6 \mathrm{~L}$ of unpasteurized whole milk twice daily while housed in individual pens until approximately 3 wk of age. Calves were then moved as a group of 20 animals per pen. Eight liters of unpasteurized whole milk was offered via an automated system in the group pens (Forster Technik, DeLaval, Peterborough, ON, Canada). Calves were allowed $3 \mathrm{~L}$ per feeding within a 3 -h period. Free choice starter was offered within the first $3 \mathrm{~d}$ of life. Water was not available until the calves reached the group pen. Individual and group pens were bedded with a sawdust base covered by a top layer of straw. Calves were removed from the group pen at approximately 8 wk of age. Calves spent the first 24 to $36 \mathrm{~h}$ of life in a straw-bedded room adjacent to the maternity area before moving to 1 of 2 identical recently built barns. Each barn held 40 calves in individual stalls and 80 calves split between 4 group pens for a total of 120 calves per barn. Each barn was curtain sided and used both natural and positive pressure ventilation systems. As calves were born, individual stalls were filled in one barn, followed by the second barn. The owners of this commercial facility were not willing to have a $\mathrm{NC}$ group; therefore, calves were only enrolled into PC and IN groups. Treatment groups were randomly assigned at the barn level before the start of the study by picking the protocol from a hat.

In herd 3 (lactating cows, $\mathrm{n}=55$ ), calves were fed 4 $\mathrm{L}$ of single source colostrum within $24 \mathrm{~h}$ of birth, fol- lowed by $2 \mathrm{~L}$ of unpasteurized whole milk 3 times daily until abrupt weaning at approximately $8 \mathrm{wk}$ of age. All calves were housed outside tethered to individual plastic hutches until weaning, at which point they were moved to group housing. Free choice water and calf starter were available beginning at $3 \mathrm{~d}$ of age. Calves were blocked by sex, and treatment was assigned to each hutch by drawing protocol names from a hat in sets of 3 without replacement.

\section{Sample Collection, Weighing, Health Scoring, and Ultrasonographic Data Collection}

For assessing passive transfer of maternal antibodies, whole blood was collected from 3- to 6-d-old calves at enrollment, by jugular venipuncture, using a 20-gauge, 1-inch $(2.54 \mathrm{~cm})$ hypodermic needle (BD Vacutainer Precision Glide, Becton, Dickinson and Co., Franklin Lakes, NJ), into sterile, plastic, commercial blood collection tubes without anticoagulant. Blood tubes were stored on ice and serum was separated by centrifugation at $1,500 \times g$ for $15 \mathrm{~min}$ at $\sim 20^{\circ} \mathrm{C}$, within 4 to 6 $\mathrm{h}$ of collection. Analysis of serum total protein (STP) was performed by a research assistant using a digital refractometer (Misco PA202X-003-105, Cleveland, $\mathrm{OH}$ ). Also at enrollment, a 1- to 2-cm skin sample (ear notch) was collected from the ventral edge of the pinna to determine the bovine viral diarrhea persistent infection status for each calf using antigen-capture ELISA. Ear notch samples were placed in sterile, plastic, commercial blood collection tubes without anticoagulant, stored on ice, and refrigerated within 4 to $6 \mathrm{~h}$ of collection before submission to the University of Guelph's Animal Health Laboratory.

At each examination, calves were weighed 3 times using a weigh tape (Coburn Company, Whitewater, WI), and these weights were averaged to provide the weight for each examination. Average daily gain during the study period was calculated by dividing the difference in the weight recorded $56 \mathrm{~d}$ after enrollment and enrollment by 56 . Calves that died before $56 \mathrm{~d}$ of age were excluded from the ADG analysis.

Respiratory scoring (Lago et al., 2006; McGuirk and Peek, 2014) and TUS were performed at each visit. Briefly, the RS assigned 0 to 3 points for each of the following categories: rectal temperature, nasal discharge, cough, and ocular discharge or ear position. Respiratory scores could range from 0 to 12 and any calf with RS $>4$ was considered sick (McGuirk and Peek, 2014). Fecal scores (FS) were obtained after each RS by direct visualization of fresh manure. Digital examination of the rectum was used to stimulate defecation. Fecal scores were based on a 3 -point scale: 1 = sample is in "patty" form; minimal water content, does not flow across or 
down a surface; $2=$ sample is more of a puddle, some water content, flows slowly across or down a surface; $3=$ sample is watery, flows across or down a surface while leaving some to no adherent material (Ollivett et al., 2009). Regarding TUS, both lungs were scanned and images were interpreted as previously described (Ollivett et al., 2015). The depth and dorsal to ventral extent of consolidated lung were measured within each intercostal space using the 1-cm grid lines on the ultrasound screen. Observations and measurements were spoken and recorded using a digital voice recorder and later manually transcribed into a database (Microsoft Access 2010, Microsoft Corp., Redmond, WA).

\section{Statistical Analyses}

In addition to the 3 treatment groups, several potential explanatory covariates were investigated. Continuous variables included age at enrollment in days (EAGE), age at first TUS examination in days, and weight at first ultrasound in kilograms (W1). Nondichotomous categorical variables included treatment (1 $=\mathrm{PC} ; 2=\mathrm{IN} ; 3=\mathrm{NC})$, herd $(1=$ herd $1 ; 2=$ herd $2 ; 3=$ herd 3 ), season of enrollment (winter $=$ January through March; spring = April through June; summer $=$ July through September), birth weight category (0 $=\mathrm{W} 1<40 \mathrm{~kg} ; 1=40 \mathrm{~kg} \leq \mathrm{W} 1<46 \mathrm{~kg} ; 2=\mathrm{W} 1 \geq$ $46 \mathrm{~kg}$ ), and exam (chronologically ordered number of TUS exam). Dichotomous variables included ILL (all $\mathrm{RS} \leq 4=0 ; 1$ or more RS $>4=1)$; CON $(<3 \mathrm{~cm}$ TUS lung consolidation on at all examinations $=0$; 1 or more TUS examinations with $\geq 3 \mathrm{~cm}$ TUS lung consolidation present $=1)$; sex $($ male $=0$; female $=$ 1); twin (no $=0$; yes $=1$ ); dystocia (DYST; calving with no assistance or easy pull $=0$, calving with hard pull or surgical delivery $=1$ ); failure of passive transfer $(\mathbf{F P T} ; \mathrm{STP} \geq 5.2 \mathrm{~g} / \mathrm{dL}=0 ; \mathrm{STP}<5.2 \mathrm{~g} / \mathrm{dL}=1$, Tyler et al., 1996); housing (outdoors $=0$; indoors $=$ 1 ); scour $(\mathrm{FS}<3$ within $21 \mathrm{~d}$ of enrollment $=0 ; \mathrm{FS}=$ 3 during at least one exam within $21 \mathrm{~d}$ of enrollment $=$ 1); and rib fracture (RIBF; rib fracture not palpable or visible on TUS $=0$; rib fracture palpable and visible on TUS = 1). The 3 -cm cut-off value for CON was determined by selecting the value between the 90 th and 95th percentiles (2.25 and $3.5 \mathrm{~cm}$, respectively) of TUS lung consolidation in those calves with any amount of lung consolidation during their first TUS examination. The scour variable was intended to capture those calves having at least one bout of severe diarrhea during the high-risk period of the first $21 \mathrm{~d}$ of life.

A standard statistical package was used for all analyses (SAS version 9.4, SAS Institute Inc., Cary, NC), except for the sample size calculation (Stata 12.1, Stata Corp. LP, College Station, TX). This study was designed as a superiority trial where IN was expected to outperform $\mathrm{NC}$ and PC. Initial expected prevalence of respiratory disease was 15 to $20 \%$. Sample size of 270 calves per treatment was estimated initially to provide a power of $80 \%$ and detect a $10 \%$ difference in clinical disease with $\alpha=0.05$. Sample size was re-estimated and adjusted to 220 calves per treatment after recognizing the higher than anticipated level of disease and presumed greater effect of the intervention. All continuous variables were assessed for normality using the Shapiro-Wilk test. Measures of central tendency are presented as mean (SD) for ADG, and median (interquartile range, IQR) for EAGE. Raw ADG were compared using the $t$-test. Enrollment ages were compared by Wilcoxon rank sum test. Categorical variables (birth weight, DYST, twin, sex, FPT, RIBF, ILL, and CON) were assessed with contingency tables and chi-squared analysis, or Fisher's exact test when individual cell counts were $<5$. Relative risk was calculated to estimate the association between RIBF and DYST and the association between ILL and CON. A kappa was calculated using ILL and CON as variables in the FREQ procedure with the TEST KAPPA statement. The purpose of this kappa test was to determine the agreement between CON and ILL in their ability to identify calves with BRD during the preweaning period. When a calf died or was euthanized due to severe disease, a field-based postmortem examination was performed by a veterinarian. Overall mortality risk was calculated by dividing the number of calves that died during the follow-up period by the total number of enrolled calves. The overall risk of death from BRD was calculated by dividing the number of calves that died as the result of BRD during the followup period by the total number of enrolled calves. The BRD case fatality rate was calculated by dividing the number of calves that died due to BRD by the total number of calves positive for BRD. Calves positive for CON or ILL were considered positive for BRD.

Logistic regression models were fit for the outcomes ILL and CON using the GLIMMIX procedure. The explanatory variable of interest was treatment. All variables that had $P<0.20$ after univariable analysis were offered into a multivariable model as fixed effects, including herd. Treatment was forced into each model. All 2-way interactions between treatment and fixed effects were tested. Manual backward stepwise elimination was used to refine the model to include variables that had $\alpha \leq 0.05$ level. Additionally, a change in estimate criterion of $\geq 25 \%$ was used to assess for confounding before the final elimination of a variable. Predicted means for CON and ADG were assessed using the LSMEANS statement and these were adjusted using the DIFF option. Type 3 tests of fixed effects were used to determine significance which was set at 
$\alpha<0.05$. Collinearity (type II tolerance $<0.10$ ) was assessed with the GLM procedure.

Average daily gain was modeled using the MIXED procedure. The primary explanatory variable of interest was treatment. Variables were offered similar to the logistic regression models. Knowing that male calves typically grow faster than heifer calves (Koch et al., 1959), sex was forced into the model even though it was not significant in the univariable analysis. Predictions based on categorical variables were assessed using the LSMEANS statement. Pearson correlation coefficients were calculated to assess the correlations between model predictions and actual observations. Each model was assessed graphically for outliers and the normality of residuals was tested using the Shapiro-Wilk, AndersonDarling, Kolmogorov-Smirnov, and Cramer-von Mises tests. Significance was set at $\alpha<0.05$.

\section{RESULTS}

A total of 468 calves from 3 herds were enrolled and randomized into 3 treatment groups $(\mathrm{PC}, \mathrm{n}=211$; IN, $\mathrm{n}=215 ; \mathrm{NC}, \mathrm{n}=42$ ). All calves had a negative bovine viral diarrhea virus persistent infection status at enroll- ment. Distributions of variables potentially affected by incomplete randomization are summarized in Table 1. A difference was observed in treatment group size between herds $(P<0.0001)$ due to the lack of NC at herd 2 . However, no difference $(P=0.48)$ was present in the proportions of calves enrolled in PC and IN. Calves were younger at enrollment in IN (median age, IQR: 4, 3-5 d) compared with PC (median age, IQR: 4, 4-5 $\mathrm{d} ; P=0.02$ ) and NC (median age, IQR: $5,4-6 \mathrm{~d} ; P=$ $0.02)$. However, no difference $(P=0.22)$ was present in EAGE between PC and NC. Serum protein data were missing from some calves in PC $(\mathrm{n}=3)$, IN $(\mathrm{n}=5)$, and NC $(\mathrm{n}=2)$. The risk of FPT was greater for NC (15\%) compared with PC (4\%) and IN $(7 \% ; P=0.01$ and $P=0.08$, respectively). The risk of FPT was not different between PC and IN $(P=0.29)$. Rib fractures were observed in $6 \%(\mathrm{n}=28)$ of calves. Rib fractures were associated with dystocia (RR 3.78, 95\% CI: 1.83 , 7.78). However, no association was observed between rib fractures and treatment $(P=1.0)$.

Overall, 54\% ( $\mathrm{n}=251)$ calves were ILL positive. Before controlling for potentially confounding explanatory variables, $\mathrm{NC}$ had a lower proportion of ILL positive calves than $\mathrm{PC}(\mathrm{NC}=33 \%$ vs. $\mathrm{PC}=59 \% ; P=0.003)$,

Table 1. Distribution of variables in 468 calves enrolled in a randomized blinded field study by calf characteristic and treatment group

\begin{tabular}{|c|c|c|c|c|c|}
\hline \multirow[b]{2}{*}{ Variable } & \multirow[b]{2}{*}{ Category } & \multicolumn{3}{|c|}{ Treatment group, no. (\% across category) } & \multirow[b]{2}{*}{$P$-value } \\
\hline & & $\mathrm{PC}^{1}$ & $\mathrm{IN}^{2}$ & $\mathrm{NC}^{3}$ & \\
\hline \multirow[t]{3}{*}{ Herd } & Herd 1 & $46(40.4)$ & $37(32.5)$ & $31(27.2)$ & \multirow[t]{3}{*}{$<0.0001$} \\
\hline & Herd 2 & $156(48.0)$ & $169(52.0)$ & - & \\
\hline & Herd 3 & $9(31.0)$ & $9(31.0)$ & $11(37.9)$ & \\
\hline \multirow[t]{3}{*}{ Birth weight (kg) } & $<40 \mathrm{~kg}$ & $50(46.7)$ & $49(45.8)$ & $8(7.5)$ & \multirow[t]{3}{*}{0.42} \\
\hline & $40-46 \mathrm{~kg}$ & $117(48.0)$ & $106(43.4)$ & $21(8.6)$ & \\
\hline & $>46 \mathrm{~kg}$ & $44(37.6)$ & $60(51.3)$ & $13(11.1)$ & \\
\hline \multirow[t]{2}{*}{ Calving ease } & Hard pull, no & $183(45.6)$ & $182(45.4)$ & $36(9.0)$ & \multirow[t]{2}{*}{0.74} \\
\hline & Hard pull, yes & $25(42.4)$ & $30(50.9)$ & $4(6.8)$ & \\
\hline \multirow[t]{2}{*}{ Twin } & No & $184(44.1)$ & $193(46.3)$ & $40(9.6)$ & \multirow[t]{2}{*}{0.58} \\
\hline & Yes & $21(50.0)$ & $19(45.2)$ & $2(4.8)$ & \\
\hline \multirow[t]{2}{*}{$\mathrm{FPT}^{4}$} & No & $200(46.5)$ & $196(45.6)$ & $34(7.9)$ & \multirow[t]{2}{*}{0.04} \\
\hline & Yes & $9(31.0)$ & $14(48.3)$ & $6(20.7)$ & \\
\hline \multirow[t]{2}{*}{ Sex } & Male & $112(44.3)$ & $124(42.3)$ & $17(6.7)$ & \multirow[t]{2}{*}{0.11} \\
\hline & Female & $99(46.1)$ & $91(42.3)$ & $25(11.6)$ & \\
\hline \multirow[t]{2}{*}{ First $\mathrm{RS}^{5}$} & $<5$ & $208(45.3)$ & $211(46.0)$ & $40(8.7)$ & \multirow[t]{2}{*}{0.33} \\
\hline & $\geq 5$ & $3(33.3)$ & $4(44.4)$ & $2(22.2)$ & \\
\hline \multirow[t]{2}{*}{ First $\mathrm{US}^{6}$} & $<3 \mathrm{~cm}$ & $207(45.0)$ & $211(45.9)$ & $42(9.1)$ & \multirow[t]{2}{*}{1.00} \\
\hline & $\geq 3 \mathrm{~cm}$ & $4(50.0)$ & $4(50.0)$ & 0 & \\
\hline
\end{tabular}

${ }^{1}$ Positive control (PC): $2 \mathrm{~mL}$ of commercially available multivalent injectable vaccine against bovine respiratory syncytial virus (BRSV), infectious bovine rhinotracheitis (IBR), parainfluenza $3\left(\mathrm{PI}_{3}\right)$, and bovine viral diarrhea administered subcutaneously at 6 wk of age.

${ }^{2}$ Intranasal vaccine (IN): $2 \mathrm{~mL}$ of commercially available trivalent injectable vaccine against BRSV, IBR, and $\mathrm{PI}_{3}$ administered intranasally at 3 to $6 \mathrm{~d}$ of age.

${ }^{3}$ Negative control (NC): $2 \mathrm{~mL}$ of sterile saline administered both intranasally and subcutaneously at 3 to $6 \mathrm{~d}$ of age and 6 wk of age.

${ }^{4}$ Failure of passive transfer; cut-off serum total protein $<5.2 \mathrm{mg} / \mathrm{dL}$.

${ }^{5}$ Respiratory score, Wisconsin Calf Scoring Chart.

${ }^{6}$ Ultrasound examination. 
Table 2. Multivariable logistic regression model for the prediction of clinical illness (ILL) in 468 Holstein dairy calves from 3 herds in southwestern Ontario randomly assigned to receive 1 of 3 preweaning vaccination protocols $^{1}$

\begin{tabular}{lclcc}
\hline Variable & Estimate & SE & $P$-value & Odds ratio (95\% CI) \\
\hline $\begin{array}{l}\text { Intercept } \\
\text { Treatment }\end{array}$ & -1.41 & 0.51 & - & - \\
$\mathrm{PC}^{2}$ & 0.15 & 0.41 & 0.71 & $1.16(0.52-2.61)$ \\
$\mathrm{IN}^{3}$ & -0.16 & 0.42 & 0.71 & $0.85(0.38-1.93)$ \\
$\mathrm{NC}^{4}$ & Referent & - & - & - \\
Sex & & & & $1.51(1.02-2.23)$ \\
$\quad$ Female & 0.41 & 0.20 & 0.04 & - \\
Male & Referent & - & - & $1.91(1.25-2.93)$ \\
Scours & 0.65 & 0.22 & $<0.01$ & - \\
Yes & Referent & - & - & $1.26(0.50-3.16)$ \\
No & & & & $4.85(1.95-12.06)$ \\
Herd & 0.23 & 0.47 & 0.62 & - \\
1 & 1.58 & 0.46 & $<0.001$ & \\
2 & Referent & - & - & \\
3 & & &
\end{tabular}

${ }^{1}$ ILL calves had a respiratory score $>4$ on at least one occasion during the study period.

${ }^{2}$ Positive control (PC): $2 \mathrm{~mL}$ of commercially available multivalent injectable vaccine against bovine respiratory syncytial virus (BRSV), infectious bovine rhinotracheitis (IBR), parainfluenza $3\left(\mathrm{PI}_{3}\right)$, and bovine viral diarrhea administered subcutaneously at 6 wk of age.

${ }^{3}$ Intranasal vaccine (IN): $2 \mathrm{~mL}$ of commercially available trivalent injectable vaccine against BRSV, IBR, and $\mathrm{PI}_{3}$ administered intranasally at 3 to $6 \mathrm{~d}$ and $6 \mathrm{wk}$ of age.

${ }^{4}$ Negative control (NC): $2 \mathrm{~mL}$ of sterile saline administered both intranasally and subcutaneously at 3 to $6 \mathrm{~d}$ of age and 6 wk of age.

and IN (IN $=53 \% ; P=0.02)$. No difference was observed in the proportion of ILL calves between PC and IN $(P=0.20)$. After controlling for the effects of herd, sex, and scour, treatment was not associated with the odds of ILL (Table 2; $P=0.32$ ).

Overall, $54 \%(\mathrm{n}=253)$ of the calves were CON positive. Before controlling for potentially confounding explanatory variables, $\mathrm{NC}$ had a lower proportion of $\mathrm{CON}$ positive calves than $\mathrm{PC}(\mathrm{NC}=33 \%$ vs. $\mathrm{PC}=60 \% ; P$ $=0.002)$, and IN $(\mathrm{IN}=53 \% ; P=0.02)$. There was no difference in proportion of CON positive calves between $\mathrm{PC}$ and IN $(P=0.14)$. However, after controlling for herd, DYST, and RIBF, treatment was significantly associated with the odds of CON (Table $3 ; P=0.005$ ). The predicted probabilities of $\mathrm{CON}$ for each treatment are shown in Figure 1. The odds of CON in PC were $1.63(95 \%$ CI: $1.04-2.56)$ times the odds of CON in IN $(P=0.03)$ and 0.38 (95\% CI: $0.16-0.93)$ times the odds of $\mathrm{CON}$ in $\mathrm{NC}(P=0.03)$. The odds of $\mathrm{CON}$ in IN were 0.23 (95\% CI: $0.09-0.59)$ times the odds of $\mathrm{CON}$ in $\mathrm{NC}$ (0.002). A $2 \times 2$ contingency table compares the overall distribution of CON and ILL (Table 4). The outcomes CON and ILL were associated (RR 2.20, 95\% CI: 1.79, $2.70 ; P<0.0001)$, but their ability to identify the same animals as affected with respiratory disease during the preweaning period was only fair (kappa $=0.38$ ).

The ADG for all calves was $0.56 \mathrm{~kg} / \mathrm{d}(\mathrm{SD}=0.17)$. Before controlling for potentially confounding explanatory variables, the ADG for $\mathrm{NC}$ was $0.69 \mathrm{~kg} / \mathrm{d}(\mathrm{SD}=$
0.14), which was greater $(P<0.0001)$ than both $\mathrm{PC}$ and IN; however, $\mathrm{PC}$ and IN were not different $[\mathrm{PC}$ : $0.55 \mathrm{~kg} / \mathrm{d}(\mathrm{SD}=0.17)$ vs. IN: $0.55 \mathrm{~kg} / \mathrm{d}(\mathrm{SD}=0.17$; $P=0.56]$. Multivariable linear regression revealed a significant interaction between treatment and herd on the outcome ADG $(P<0.01$; Table 5, Figure 2). In herd 1 , IN decreased ADG by $0.10 \mathrm{~kg} / \mathrm{d}(\mathrm{SE}=0.03)$ compared with PC $(P<0.01)$. In contrast, at herd 2 , IN increased ADG by $0.03 \mathrm{~kg} / \mathrm{d}(\mathrm{SE}=0.02 ; P=$ $0.04)$. Treatment did not influence ADG at herd $3(P>$ $0.25)$. Thirty-nine $(8 \%)$ calves died or were euthanized during the study. The overall risk of death from respiratory disease was $1.9 \%(\mathrm{n}=9)$. The case fatality rate for calves that were positive for CON or ILL was $3 \%$ (9/301). No difference was observed in overall mortality rates between vaccine protocols $(P=0.58)$.

\section{DISCUSSION}

The purpose of this study was to compare the effect of an intranasal vaccine, given to 3 - to 6 -d-old calves, with a subcutaneous vaccine, given later in life, and a placebo vaccine. Two different classification methods were used to identify diseased calves, and they included RS and TUS. Growth was assessed during the first $56 \mathrm{~d}$ of life. Two doses of a commercially available trivalent IN respiratory vaccine (experimental treatment, IN, first dose: 3 to 6 d of age; second dose: 6 wk of age) had no effect on the odds of having at least one episode of 
Table 3. Multivariable logistic regression model for the prediction of CON in 451 Holstein dairy calves from 3 herds in southwestern Ontario randomly assigned to receive 1 of 3 preweaning vaccination protocols ${ }^{1}$

\begin{tabular}{|c|c|c|c|c|}
\hline Variable & Estimate & SE & $P$-value & Odds ratio $(95 \% \mathrm{CI})$ \\
\hline$\overline{\text { Intercept }}$ & -0.79 & 0.54 & - & - \\
\hline \multicolumn{5}{|l|}{ Treatment } \\
\hline $\mathrm{PC}^{2}$ & -0.96 & 0.46 & 0.03 & $0.38(0.16-0.93)$ \\
\hline $\mathrm{IN}^{3}$ & -1.45 & 0.47 & $<0.01$ & $0.23(0.09-0.59)$ \\
\hline $\mathrm{NC}^{4}$ & Referent & - & -0.01 & $0.20(0.050 .03)$ \\
\hline \multicolumn{5}{|l|}{ Dystocia } \\
\hline Yes & 0.73 & 0.35 & 0.04 & $2.08(1.05-4.15)$ \\
\hline No & Referent & - & - & - \\
\hline \multicolumn{5}{|c|}{ Rib fracture } \\
\hline Yes & 1.31 & 0.56 & 0.02 & $3.71(1.23-11.17)$ \\
\hline No & Referent & - & - & - \\
\hline \multicolumn{5}{|l|}{ Herd } \\
\hline 1 & 0.16 & 0.57 & 0.77 & $1.17(0.39-3.56)$ \\
\hline 2 & 2.64 & 0.58 & $<0.0001$ & $14(4.4-43.82)$ \\
\hline 3 & Referent & - & - & - \\
\hline
\end{tabular}

${ }^{1} \mathrm{CON}$ calves had $\geq 3 \mathrm{~cm}$ lung consolidation on at least one occasion throughout the study period.

${ }^{2}$ Positive control (PC): $2 \mathrm{~mL}$ of commercially available multivalent injectable vaccine against bovine respiratory syncytial virus (BRSV), infectious bovine rhinotracheitis (IBR), parainfluenza $3\left(\mathrm{PI}_{3}\right)$, and bovine viral diarrhea administered subcutaneously at $6 \mathrm{wk}$ of age.

${ }^{3}$ Intranasal vaccine (IN): $2 \mathrm{~mL}$ of commercially available trivalent injectable vaccine against BRSV, IBR, and $\mathrm{PI}_{3}$ administered intranasally at 3 to $6 \mathrm{~d}$ and $6 \mathrm{wk}$ of age.

${ }^{4}$ Negative control (NC): $2 \mathrm{~mL}$ of sterile saline administered both intranasally and subcutaneously at 3 to $6 \mathrm{~d}$ of age and 6 wk of age.

ILL during the study period, compared with a commercially available subcutaneous product (PC, only dose: 6 wk of age) or sterile saline (NC) after accounting for potentially confounding variables. In contrast, IN significantly reduced the odds of having at least one episode of CON compared with both positive and negative controls. The effect of IN on calf growth was herd dependent. This interaction resulted in a greater ADG in herd 2 in IN treated calves compared with PC. In

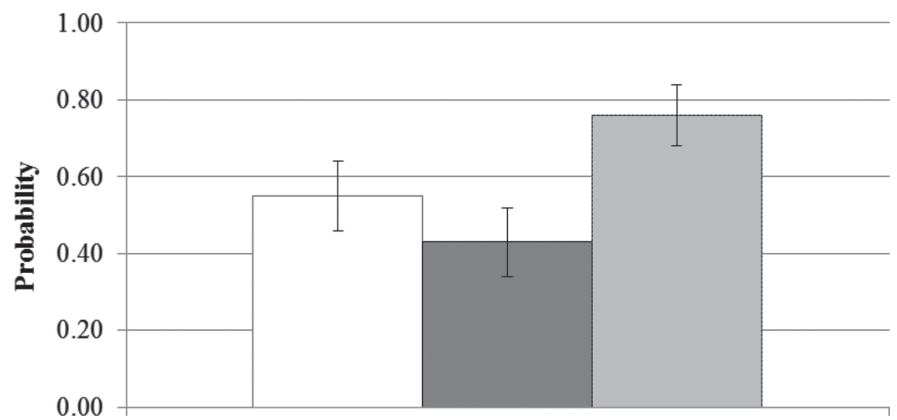

Figure 1. Predicted probability of $\mathrm{CON}$ by vaccine protocol after controlling for herd, dystocia, and rib fractures. Error bars represent SEM. CON $=$ occurrence of $\geq 3 \mathrm{~cm}$ lung consolidation at least once in the study period. $\mathrm{PC}=$ white; $\mathrm{IN}=$ dark gray; $\mathrm{NC}=$ light gray. $\mathrm{PC}=$ positive control: $2 \mathrm{~mL}$ of commercially available multivalent injectable vaccine against bovine respiratory syncytial virus (BRSV), infectious bovine rhinotracheitis (IBR), parainfluenza $3\left(\mathrm{PI}_{3}\right)$, and bovine viral diarrhea administered subcutaneously at 6 wk of age. IN = intranasal treatment: $2 \mathrm{~mL}$ of commercially available trivalent injectable vaccine against BRSV, IBR, and $\mathrm{PI}_{3}$ administered intranasally at 3 to $6 \mathrm{~d}$ and 6 wk of age. $\mathrm{NC}=$ negative control: $2 \mathrm{~mL}$ of sterile saline administered both intranasally and subcutaneously at 3 to $6 \mathrm{~d}$ and $6 \mathrm{wk}$ of age. herd 1, IN was associated with either a lower ADG or no change in ADG compared with the $\mathrm{PC}$ and $\mathrm{NC}$, respectively. No differences in ADG were observed in herd 3.

Clinical observation is used commonly to detect respiratory disease (Amrine et al., 2013). However, compared with TUS, respiratory scoring did not detect the disease sparing effects of IN in the current study. As previously mentioned, lung lesions typically affect more calves at slaughter than indicated by observation-based treatment records (Wittum et al., 1996; Thompson et al., 2006), suggesting the presence of subclinical disease. A study done in a single herd demonstrated that clinical signs alone failed to identify lung lesions, which were seen in more than $60 \%$ of preweaned calves (Ollivett and Buczinski, 2016). Recent findings demonstrate that lung consolidation has long-term implications on dairy calf survival and reproduction (Adams and Buczinski, 2016; Teixeira et al., 2017). This highlights

Table 4. A $2 \times 2$ contingency table comparing the distribution of CON and ILL in Holstein dairy calves in Southwestern Ontario, enrolled in a randomized vaccine field trial $(n=468)$

\begin{tabular}{lrr}
\hline & \multicolumn{2}{c}{$\mathrm{CON}^{2}$} \\
\cline { 2 - 3 } $\mathrm{ILL}^{1}$ & 1 & 0 \\
\hline 1 & 181 & 70 \\
0 & 72 & 145 \\
\hline
\end{tabular}

${ }^{1}$ At least one respiratory score $>4$.

${ }^{2}$ At least one ultrasound exam with $\geq 3 \mathrm{~cm}$ lung consolidation. 
Table 5. Multivariable linear regression model for ADG on 421 Holstein dairy calves from 3 herds in southwestern Ontario, randomly assigned to receive 1 of 3 preweaning vaccination protocols ${ }^{1}$

\begin{tabular}{|c|c|c|c|c|}
\hline Variable & Estimate & $\mathrm{SE}$ & $95 \% \mathrm{CI}$ & $P$-value \\
\hline Intercept & 0.513 & 0.0761 & $0.364,0.663$ & $<0.0001$ \\
\hline \multicolumn{5}{|l|}{ Treatment } \\
\hline $\mathrm{PC}^{2}$ & 0.00439 & 0.0638 & $-0.121,0.130$ & 0.95 \\
\hline $\mathrm{IN}^{3}$ & -0.0426 & 0.0655 & $-0.171,0.0862$ & 0.52 \\
\hline $\mathrm{NC}^{4}$ & Referent & - & - & - \\
\hline \multicolumn{5}{|l|}{ Twin } \\
\hline Yes & -0.1380 & 0.0253 & $-0.188,-0.0882$ & $<0.0001$ \\
\hline No & Referent & - & - & - \\
\hline \multicolumn{5}{|l|}{ Dystocia } \\
\hline Yes & -0.0467 & 0.0212 & $-0.0884,-0.00497$ & 0.028 \\
\hline No & Referent & - & - & - \\
\hline \multicolumn{5}{|l|}{ House } \\
\hline Outdoors & -0.0285 & 0.0378 & $-0.103,0.0459$ & 0.45 \\
\hline Indoors & Referent & - & - & - \\
\hline \multicolumn{5}{|l|}{ Rib fracture } \\
\hline Yes & -0.0565 & 0.0285 & $-0.113,-0.00062$ & 0.048 \\
\hline No & Referent & - & - & - \\
\hline \multicolumn{5}{|l|}{ Sex } \\
\hline Male & 0.0447 & 0.0143 & $0.0166,0.0728$ & $<0.01$ \\
\hline Female & Referent & - & - & - \\
\hline \multicolumn{5}{|l|}{ Birth weight } \\
\hline$<40 \mathrm{~kg}$ & -0.0645 & 0.0212 & $-0.106,-0.0228$ & $<0.01$ \\
\hline $40-46 \mathrm{~kg}$ & -0.0217 & 0.0173 & $-0.0557,0.0123$ & 0.21 \\
\hline$>46 \mathrm{~kg}$ & Referent & - & - & - \\
\hline Enrollment age (d) & 0.00332 & 0.0060 & $-0.0086,0.0152$ & 0.58 \\
\hline \multicolumn{5}{|c|}{ Protocol $\times$ herd interaction } \\
\hline $\mathrm{PC} \times 1$ & 0.0494 & 0.0733 & $-0.0947,0.193$ & 0.50 \\
\hline $\mathrm{PC} \times 2$ & -0.0804 & 0.0687 & $-0.216,0.0547$ & 0.4 \\
\hline $\mathrm{IN} \times 1$ & 0.00023 & 0.0754 & $-0.149,0.148$ & 0.99 \\
\hline \multicolumn{5}{|l|}{ Herd } \\
\hline 1 & -0.0549 & 0.0606 & $-0.174,0.0642$ & 0.37 \\
\hline 2 & -0.173 & 0.0625 & $-0.295,-0.0497$ & 0.006 \\
\hline 3 & Referent & - & - & - \\
\hline
\end{tabular}

${ }^{1}$ Calves dying before $56 \mathrm{~d}$ of age were not included in the analysis.

${ }^{2}$ Positive control (PC): $2 \mathrm{~mL}$ of commercially available multivalent injectable vaccine against bovine respiratory syncytial virus (BRSV), infectious bovine rhinotracheitis (IBR), parainfluenza $3\left(\mathrm{PI}_{3}\right)$, and bovine viral diarrhea administered subcutaneously at 6 wk of age.

${ }^{3}$ Intranasal vaccine (IN): $2 \mathrm{~mL}$ of commercially available trivalent injectable vaccine against BRSV, IBR, and $\mathrm{PI}_{3}$ administered intranasally at 3 to $6 \mathrm{~d}$ and $6 \mathrm{wk}$ of age.

${ }^{4}$ Negative control (NC): $2 \mathrm{~mL}$ of sterile saline administered both intranasally and subcutaneously at 3 to $6 \mathrm{~d}$ of age and 6 wk of age.

the importance of minimizing the incidence of $\mathrm{CON}$ in preweaned dairy calves. The use of IN may prove beneficial in minimizing the risk of $\mathrm{CON}$, given the findings of the present study.

The fact that agreement between diagnostic methods was just fair indicates that different populations of calves were identified by each predictor. It is possible that a clinical difference did not exist, the cut-points used to create ILL and CON were inappropriate, or the subjective nature and variability inherent to clinical scoring precluded finding small differences in a limited study population. The disagreement between the 2 variables rested evenly between CON positive calves that were ILL negative and ILL positive calves that were CON negative. This could be the result of clinical scoring systems that are not highly specific to the disease they are supposed to be detecting. As can be seen in the logistic regression model, the significant association between scour and ILL highlights the fact diseases other than BRD can evoke a positive test when respiratory scoring. The effect of these variables on objective measures, such as calf growth, will help researchers understand which variable most accurately identifies the population of calves affected with BRD.

Rib fractures are common in neonatal foals (Jean et al., 1999); however, their presence and effect on ADG was an unexpected finding in this study in dairy calves. Rib fractures were typically located near the costochondral junction of the cranial thorax, similar to previous reports (Jean et al., 1999), and were easily imaged via TUS. Six percent of calves in the current study had obvious rib fractures, which is much lower than previous reports of $23 \%$ in calves that die during the perinatal period (Schuijt, 1990) and $40 \%$ of calves 


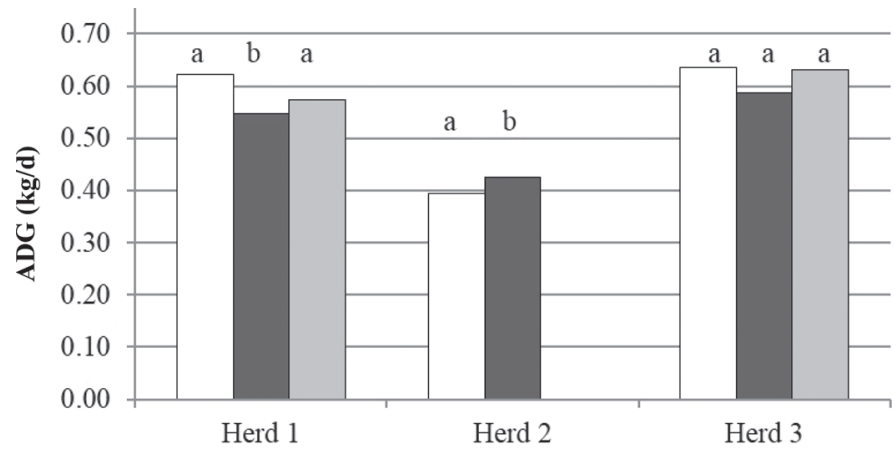

Figure 2. Differences in the LSM of ADG $(\mathrm{kg} / \mathrm{d})$ showing the interaction between treatment and herd. The linear mixed model controlled for vaccine type, herd, twin, housing, dystocia, rib fractures, birth weight, and age at enrollment. Different letters (a,b) within a herd category are statistically different $(P<0.05) . \mathrm{PC}=$ white; $\mathrm{IN}=$ dark gray; $\mathrm{NC}=$ light gray. $\mathrm{PC}=$ positive control: $2 \mathrm{~mL}$ of commercially available multivalent injectable vaccine against bovine respiratory syncytial virus (BRSV), infectious bovine rhinotracheitis (IBR), parainfluenza $3\left(\mathrm{PI}_{3}\right)$, and bovine viral diarrhea administered subcutaneously at $6 \mathrm{wk}$ of age. IN = intranasal treatment: $2 \mathrm{~mL}$ of commercially available trivalent injectable vaccine against BRSV, IBR, and $\mathrm{PI}_{3}$ administered intranasally at 3 to $6 \mathrm{~d}$ and 6 wh of age. $\mathrm{NC}=$ negative control: $2 \mathrm{~mL}$ of sterile saline administered both intranasally and subcutaneously at 3 to $6 \mathrm{~d}$ and 6 wk of age.

born with veterinary assistance (Mee, 2008). This likely reflects different study populations, although an equine study reported a $20 \%$ prevalence of rib fractures in foals in one breeding herd, twice as many resulting from dystocia than from normal parturitions (Jean et al., 1999). Despite the fact that none of the observed fractures were severely displaced or causing internal thoracic abnormalities, such as hemothorax or pneumothorax, rib fractures did contribute significantly to the variation in ADG. Interestingly, recent work has shown that anti-inflammatory doses of meloxicam given immediately after a difficult birth results in greater calf growth during the early weeks of life (Murray, 2014). One reason for this finding could be treatment of the pain and inflammation associated with rib fractures, which allows for improved utilization of liquid feed and greater growth. In the current study, an association was present between dystocia and rib fractures; however, the correlation was very low and highlights the fact that a particularly difficult calving is not a prerequisite for rib fractures.

Although herd differences were noted in this study, the fact that only 3 herds were included is a limitation to the ability to draw inferences about herd specific factors regarding the efficacy of IN. Inclusion of a large number of herds varying in locale and management practices would be necessary to determine exactly which situations IN would be most effective. An additional limitation is the potential confounding effect of barn on the relationship between the measured outcomes and treatment at herd 2. This design was intentional as IN and PC calves needed to be housed separately to prevent exposure of $\mathrm{PC}$ to IN vaccine virus. The authors do acknowledge that switching the treatment status of each barn would have been ideal. However, the feasibility and additional time requirements necessary for incorporating washout periods when transitioning between groups were considered impractical. The 2 barns were close in proximity to each other, being separated by a milk preparation room; and barn age, design, flow of animals, cleaning, and labor were identical. These factors should have reduced the risk of a barn effect on calf health and performance. The authors also acknowledge that our study design was not perfectly balanced due to incomplete randomization of calves in herd 2 , resulting in a quasi-randomized clinical trial.

This study differed from previous work on the efficacy of intranasal vaccination in several ways. The calves in the current study were conventionally raised and likely exposed to chronic, low level natural challenges from the whole spectrum of respiratory pathogens (Gorden and Plummer, 2010). In contrast, previous studies incorporated controlled experimental BRSV challenges (Woolums et al., 2004; Ellis et al., 2007, 2010, 2013; Vangeel et al., 2007) and $\mathrm{PI}_{3}$ (Vangeel et al., 2007) intended to replicate natural disease in small group of animals. Despite the high prevalence of disease in the study population, the risk of death from respiratory disease was low. This suggests that the natural challenge in this study might not be as acutely aggressive as those demonstrated by single pathogen challenge models in past studies, as several calves from each BRSV challenge died or required euthanasia due to the severity of disease (Woolums et al., 2004; Ellis et al., 2007, 2013). Acknowledging that previous study designs were in part due to federal regulations regarding licensing procedures for new vaccines (Ellis et al., 2013), data from this field study may be more relevant to dairy producers and bovine practitioners.

\section{CONCLUSIONS}

A commercially available trivalent IN vaccine has the potential to reduce the lung lesions associated with BRD and improve growth in young dairy cattle. Although these findings were meaningful, herd factors played a role in determining whether or not significant changes in ADG were seen. Also, IN vaccination did not eliminate the risk of disease in the current study; therefore, this practice should not be viewed as a panacea. Best management practices regarding colostrum management, calf nutrition, housing strategies, ventila- 
tion, and appropriate vaccination protocols should be integrated to provide the optimal environment for the growing dairy calf.

\section{ACKNOWLEDGMENTS}

Financial support for this study was provided by the Ontario Ministry of Agriculture, Food, and Rural Affairs and Zoetis Canada Inc. (Kirkland, Quebec). Special thanks go to the dedicated research technicians Jolene Cyples, Jessica Cyples, Melissa Wagner, Vivianne Bielmann, Sam Deelen, Brittany Todd, Patrick Chung, and Sarah Stanger-Guy (University of Guelph, Guelph, Ontario, Canada) for their tireless efforts, and especially the dairy producers for all of the kindness shown to us during the study period.

\section{REFERENCES}

Adams, E. A., and S. Buczinski. 2016. Short communication: Ultrasonographic assessment of lung consolidation postweaning and survival to the first lactation in dairy heifers. J. Dairy Sci. 99:14651470.

Amrine, D. E., B. J. White, R. Larson, D. E. Anderson, D. A. Mosier, and N. Cernicchiaro. 2013. Precision and accuracy of clinical illness scores, compared with pulmonary consolidation scores, in Holstein calves with experimentally induced Mycoplasma bovis pneumonia. Am. J. Vet. Res. 74:310-315.

Buczinski, S., T. L. Ollivett, and N. Dendukuri. 2015. Bayesian estimation of the accuracy of the calf respiratory scoring chart and ultrasonography for the diagnosis of bovine respiratory disease in pre-weaned dairy calves. Prev. Vet. Med. 119:227-231.

Ellis, J., S. Gow, K. West, C. Waldner, C. Rhodes, G. Mutwiri, and H. Rosenberg. 2007. Response of calves to challenge exposure with virulent bovine respiratory syncytial virus following intranasal administration of vaccines formulated for parenteral administration. J. Am. Vet. Med. Assoc. 230:233-243.

Ellis, J. A., S. P. Gow, and N. Goji. 2010. Response to experimentally induced infection with bovine respiratory syncytial virus following intranasal vaccination of seropositive and seronegative calves. J. Am. Vet. Med. Assoc. 236:991-999.

Ellis, J. A., S. P. Gow, S. Mahan, and R. Leyh. 2013. Duration of immunity to experimental infection with bovine respiratory syncytial virus following intranasal vaccination of young passively immune calves. J. Am. Vet. Med. Assoc. 243:1602-1608.

Faber, S., N. Faber, T. McCauley, and R. Ax. 2005. Case study: Effects of colostrum ingestion on lactational performance. Prof. Anim. Sci. 21:420-425.

Gorden, P. J., and P. Plummer. 2010. Control, management, and prevention of bovine respiratory disease in dairy calves and cows. Vet. Clin. North Am. Food Anim. Pract. 26:243-259.

Hill, K. L., B. D. Hunsaker, H. G. Townsend, and P. J. Griebel. 2012 Mucosal immune response in newborn Holstein calves that had maternally derived antibodies and were vaccinated with an intranasal multivalent modified-live virus vaccine. J. Am. Vet. Med. Assoc. 240:1231-1240.

Jean, D., S. Laverty, J. Halley, D. Hannigan, and R. Leveille. 1999. Thoracic trauma in newborn foals. Equine Vet. J. 31:149-152.

Koch, R. M., K. E. Gregory, J. E. Ingalls, and R. L. Arthaud. 1959. Evaluating the influence of sex on birth weight and preweaning gain in beef cattle. J. Anim. Sci. 18:738-744.

Lago, A., S. McGuirk, T. Bennett, N. Cook, and K. Nordlund. 2006. Calf respiratory disease and pen microenvironments in naturally ventilated calf barns in winter. J. Dairy Sci. 89:4014-4025.
McGuirk, S. M., and S. F. Peek. 2014. Timely diagnosis of dairy calf respiratory disease using a standardized scoring system. Anim. Health Res. Rev. 15:145-147.

Mee, J. F. 2008. Newborn dairy calf management. Vet. Clin. North Am. Food Anim. Pract. 24:1-17.

Menanteau-Horta, A. M., T. R. Ames, D. W. Johnson, and J. C. Meiske. 1985. Effect of maternal antibody upon vaccination with infectious bovine rhinotracheitis and bovine virus diarrhea vaccines. Can. J. Comp. Med. 49:10-14.

Murray, C. F. 2014. Characteristics, risk factors and management programs for vitality of newborn dairy calves. PhD Thesis. Department of Population Medicine, University of Guelph, Ontario, Canada.

Ollivett, T., J. Hewson, R. Schubotz, and J. Caswell. 2013. Ultrasonographic progression of lung consolidation after experimental infection with Mannheimia haemolytica in Holstein calves. J. Vet. Intern. Med. 27:673.

Ollivett, T. L., and S. Buczinski. 2016. On-Farm use of ultrasonography for bovine respiratory disease. Vet. Clin. North Am. Food Anim. Pract. 32:19-35.

Ollivett, T. L., J. L. Caswell, D. V. Nydam, T. Duffield, K. E. Leslie, J. Hewson, and D. Kelton. 2015. Thoracic ultrasonography and bronchoalveolar lavage fluid analysis in Holstein calves affected with subclinical lung lesions. J. Vet. Intern. Med. 29:1728-1734.

Ollivett, T. L., D. V. Nydam, D. D. Bowman, J. A. Zambriski, M. L. Bellosa, T. C. Linden, and T. J. Divers. 2009. Effect of nitazoxanide on cryptosporidiosis in experimentally infected neonatal dairy calves. J. Dairy Sci. 92:1643-1648.

Rabeling, B., J. Rehage, D. Döpfer, and H. Scholz. 1998. Ultrasonographic findings in calves with respiratory disease. Vet. Rec. 143:468-471.

Schuijt, G. 1990. Iatrogenic fractures of ribs and vertebrae during delivery in perinatally dying calves: 235 cases (1978-1988). J. Am Vet. Med. Assoc. 197:1196-1202.

Teixeira, A. G. V., J. A. A. Mcart, and R. C. Bicalho. 2017. Thoracic ultrasound assessment of lung consolidation at weaning in Holstein dairy heifers: Reproductive performance and survival. J. Dairy Sci. 100:2985-2991.

Thompson, P. N., A. Stone, and W. A. Schultheiss. 2006. Use of treatment records and lung lesion scoring to estimate the effect of respiratory disease on growth during early and late finishing periods in South African feedlot cattle. J. Anim. Sci. 84:488-498.

Tizard, I. 2013. Veterinary Immunology. 9th ed. Elsevier, St. Louis, MO.

Tyler, J. W., D. D. Hancock, S. M. Parish, D. E. Rea, T. E. Besser, S. G. Sanders, and L. K. Wilson. 1996. Evaluation of 3 assays for failure of passive transfer in calves. J. Vet. Intern. Med. 10:304-307.

Vangeel, I., A. F. Antonis, M. Fluess, L. Riegler, A. R. Peters, and S. S. Harmeyer. 2007. Efficacy of a modified live intranasal bovine respiratory syncytial virus vaccine in 3-week-old calves experimentally challenged with BRSV. Vet. J. 174:627-635.

White, B. J., and D. G. Renter. 2009. Bayesian estimation of the performance of using clinical observations and harvest lung lesions for diagnosing bovine respiratory disease in post-weaned beef calves. J. Vet. Diagn. Invest. 21:446-453.

Windeyer, M. C., K. E. Leslie, S. M. Godden, D. C. Hodgins, K. D. Lissemore, and S. J. LeBlanc. 2014. Factors associated with morbidity, mortality, and growth of dairy heifer calves up to 3 months of age. Prev. Vet. Med. 113:231-240.

Wittum, T., N. Woollen, L. Perino, and E. Littledike. 1996. Relationships among treatment for respiratory tract disease, pulmonary lesions evident at slaughter, and rate of weight gain in feedlot cattle. J. Am. Vet. Med. Assoc. 209:814-818.

Woolums, A. R., C. C. Brown, J. C. Brown Jr., D. J. Cole, M. A. Scott, S. M. Williams, and C. Miao. 2004. Effects of a single intranasal dose of modified-live bovine respiratory syncytial virus vaccine on resistance to subsequent viral challenge in calves. Am. J. Vet. Res. 65:363-372. 